\title{
Cell-Intrinsic Innate Immune Control of West Nile Virus Infection
}

\author{
Michael S. Diamond ${ }^{1,2,3}$ and Michael Gale Jr. ${ }^{4}$ \\ ${ }^{1}$ Department of Molecular Microbiology, Washington University School of Medicine, St Louis. MO \\ 63110. \\ ${ }^{2}$ Department of Medicine, Washington University School of Medicine, St Louis. MO 63110. \\ ${ }^{3}$ Department of Pathology \& Immunology, Washington University School of Medicine, St Louis. \\ MO 63110. \\ ${ }^{4}$ Department of Immunology, University of Washington School of Medicine, Seattle, WA \\ 98195-7650.
}

\begin{abstract}
West Nile virus (WNV) is an enveloped positive-stranded RNA virus that has emerged over the last decade in North America to cause epidemics of meningitis, encephalitis, and acute flaccid paralysis in humans. WNV has broad species specificity, and replicates efficiently in many cell types, including those of the innate immune and central nervous systems. Recent studies have defined the pathogen recognition receptor and signaling pathways by which WNV is detected, and several effector mechanisms that contribute to protective cell-intrinsic immunity. This review focuses on recent advances in identifying the host sensors that detect WNV, the adaptor molecules and signaling pathways that regulate the induction of IFN-dependent defenses, and the proteins that limit WNV replication, spread, and disease pathogenesis.
\end{abstract}

\section{Innate Host Defense to WNV Infection}

To survive virus infection, the host must recognize invasion and develop an effective antiviral immune response. This response is initiated in infected cells after detection of nonself pathogen-associated molecular patterns (PAMPs). These PAMP motifs are detected by specific host pathogen recognition receptors (PRR), which trigger signaling cascades that induce the activation of latent transcription factors, including interferon regulatory factors (IRFs) and NF- $\mathrm{kB}$ leading to expression of virus-responsive genes, including type I interferons (IFN- $\alpha$ and $\beta$ ) and hundreds of different interferon-stimulated effector genes (ISGs). ISG products include antiviral effector molecules and immunomodulatory cytokines that serve to restrict virus replication and modulate the adaptive immune response.

West Nile virus (WNV) is a single-stranded positive-polarity RNA Flavivirus and is closely related to other human pathogens including Dengue, yellow fever, Japanese encephalitis (JEV), and tick borne encephalitis viruses. WNV cycles in nature between mosquitoes and birds with some mammalian species, including horses and humans, becoming incidentally

\section{(C) 2012 Elsevier Ltd. All rights reserved.}

Corresponding authors: Michael S. Diamond, M.D., PhD, Department of Medicine, Washington University School of Medicine, 660 S. Euclid Ave, St Louis MO 63110, diamond @ borcim.wustl.edu. Michael Gale, Jr. Department of Immunology, 959 NE Pacific Street, H-578 Health Sciences, University of Washington School of Medicine ${ }^{4}$, Seattle, WA 98195-7650. mgale@u.washington.edu. Publisher's Disclaimer: This is a PDF file of an unedited manuscript that has been accepted for publication. As a service to our customers we are providing this early version of the manuscript. The manuscript will undergo copyediting, typesetting, and review of the resulting proof before it is published in its final citable form. Please note that during the production process errors may be discovered which could affect the content, and all legal disclaimers that apply to the journal pertain. 
infected and developing disease. While most human infections remain asymptomatic or develop a mild illness, a small subset $(<1 \%)$ progress to severe neurological syndromes that include meningitis, acute flaccid paralysis, and encephalitis. Although veterinary vaccines are available commercially, there is currently no approved vaccine or therapy for WNV infection in humans. The expansion of WNV disease globally highlights a need for greater understanding of mechanisms of immune control, including the cell-intrinsic processes that restrict infection.

An effective host defense against WNV requires the antiviral actions of type I IFN. Mice lacking the common type I IFN receptor (Ifnar ${ }^{-1-}$ ) exhibit 100\% mortality, accelerated spread, and expanded cellular and tissue tropism after infection with a virulent WNV isolate [1], and infection with attenuated WNV strains results in enhanced replication and pathogenesis [2-7]. The virulence of WNV strains has been linked directly to their ability to antagonize IFN signaling $[2,6,8,9]$.

Studies by several groups have begun to define the pathogen recognition receptor and signaling pathways by which WNV and other flaviviruses are detected, and the effector mechanisms that contribute to protective cell-intrinsic immunity. These studies reveal important insights into PRR function, PAMP discrimination, and intracellular signaling pathways among different cell types relevant to WNV infection. This review will describe the recent advances that have been made in defining the host sensors that detect WNV, the signaling pathways and genes that regulate induction of IFN-dependent and -independent responses, and the proteins that limit WNV replication and disease pathogenesis.

\section{Innate immune cells controlling WNV are also targets of infection}

\section{Macrophages}

Emerging data suggests that macrophages play key roles in orchestrating control of WNV infection. Macrophages can limit infection though direct viral clearance, enhanced antigen presentation to $\mathrm{B}$ and $\mathrm{T}$ cells, and production of pro-inflammatory or antiviral cytokines and chemokines. The protective role of macrophages is highlighted by studies in mice, which demonstrated exacerbated WNV disease after selective macrophage depletion [10,11]. Macrophages have been reported to control infection of other flaviviruses (e.g., JEV) directly through the production of nitric oxide (NO) and other reactive oxygen intermediates $[12,13]$, although this mechanism has not yet been confirmed for WNV. Activation of macrophages in response to WNV infection also promotes release of type I IFN, TNF-a, IL-1 $\beta$, IL- 8 , and other cytokines, some of which have antiviral activity and reduce viral replication, at least in culture [14]. Despite their protective role in initiating innate immune defenses, macrophages also are targets of WNV $[15,16]$.

\section{Dendritic cells}

Plasmacytoid (pDC) and myeloid (mDCs) DC subsets can be distinguished based on function and surface markers. pDC lack phagocytic capacity, are less efficient in capturing and presenting antigens to T cells, but produce high levels of type I IFN in the presence of viruses or bacteria, and are thus considered to have a crucial role in antiviral immunity [17]. Low levels of WNV replication are observed in $\mathrm{pDC}$, but pro-inflammatory cytokines are produced rapidly and can accumulate to high levels. This cytokine response was not dependent on viral replication, but instead on endosomal TLR7, and could be induced by purified flavivirus virions RNA $[18,19]$. Interestingly, the host origin of WNV influences the $\mathrm{pDC}$ response, as WNV propagated in mammalian cells was a more potent inducer of IFN- $a$ secretion in pDCs whereas pDCs failed to produce IFN- $a$ when exposed to WNV grown in mosquito cells [20]. mDCs reside and circulate throughout the body, enabling them to transport antigens from peripheral sites of infection to lymphoid tissues. As professional 
antigen-presenting cells, they transmit incoming infectious signals to B and T cells, to orchestrate rapid adaptive immune responses [21]. Compared to pDCs, mDC are more readily infected by WNV and consequently, thought to contribute both to viral spread and early immune system activation. For example, WNV efficiently infects mDCs ex vivo and induces a robust type I IFN and pro-inflammatory cytokine response, which may be compromised during aging $[20,22,23]$. Despite an accumulating wealth of data on WNV infection of purified mDC ex vivo, few studies have assessed their direct function in vivo. One recent report showed that selective genetic deletion of $\mathrm{CD} 8 \mathrm{a}^{+} \mathrm{mDC}$ resulted in defective cross-presentation and virus-specific $\mathrm{CD}^{+} \mathrm{T}$ cell responses to WNV [24]. In comparison, in the skin, different inflammatory DC subsets may be responsible for initiating immune responses against WNV, including myeloid cells that are rapidly mobilized from the bone marrow [25].

\section{Pattern recognition receptor detection of WNV}

Two major classes of PRRs have been identified in mammalian cells that recognize positivestrand RNA viruses, such as WNV: 1) the toll-like receptors (TLRs); and 2) the retinoic acid-inducible gene I (RIG-I)-like receptors (RLRs). These PRR sense virus infections by recognizing nucleic acid PAMPs associated with viral RNA. Members of the RLR (RIG-I and MDA5) and TLR (TLR3 and TLR7) families are the dominant PRR that detect WNV infection.

\section{Cytosolic RIG-I-like receptors}

Retinoic acid inducible gene-I (RIG-I), and melanoma differentiation antigen 5 (MDA5) are cytoplasmic RNA helicases that recognize viral RNA products and induce transcription factor activation and type I IFN gene expression. Both RIG-I and MDA5 are composed of helicase domains at the $\mathrm{C}$-terminus and tandem caspase-activation and recruitment domains (CARD) at the N-terminus. RIG-I possesses a C-terminal repressor domain, which regulates its activity. RIG-I and MDA5 bind unique PAMPs. RIG-I ligands include single-stranded (ss)RNA containing a $5^{\prime}$-ppp, short double-stranded (ds)RNA, and uridine- or adenosinerich viral RNA motifs [26-28] whereas MDA5 binds short or long dsRNA structures although the particular motif is not well delineated [29].

Binding of viral RNA PAMP ligands by RIG-I and MDA5 induces CARD-CARD interactions with the mitochondrial-associated IFN promoter stimulator-1 (IPS-1) (also known as MAVS, VISA or CARDIF). RIG-I signaling activation is dependent on RNA PAMP binding and requires ubiquitination by TRIM25 [30], oligomerization [31], and IPS-1 multimerization [32] on mitochondrial-associated-endoplasmic reticulum membranes [33] and peroxisomes [34]. The proteins caspase-12 and 14-3-3-ع positively modulate RLR signaling of type I IFN production by regulating TRIM25-mediated ubiquitination of RIG-I $[35,36]$. Activation of IPS- 1 then promotes activation of transcription factors through interactions with TRAF3, TBK1, I $\kappa-\varepsilon$, and NEMO, driving a "signalosome" that initiates the antiviral response (Figure 1).

The precise mechanisms through which RIG-I and MDA5 recognize WNV, and the PAMPs involved in this process, remain unknown. Both RIG-I and MDA5, however, are required for optimal antiviral responses against WNV [37]. Ddx 58 (RIG-I $)^{-/-}$murine embryonic fibroblasts (MEF) support increased WNV replication and reduced IRF-3 activation, with a delayed IFN and ISG response [38, 39]. In epithelial cells, a deficiency of both RIG-I and MDA5 was required for enhanced WNV or DENV infection, as loss of either gene alone had minimal phenotypes [39]. The precise hierarchy of function of different RLR in controlling WNV infection remains an area of active study, although RIG-I may prime the early response with both RIG-I and MDA5 together regulating the response at later time 
points of infection. Of note, infection of $D d \times 58^{-1-}$ but not $M d a 5^{-1-}$ mice with JEV resulted in enhanced mortality [40]. In comparison, Mavs (IPS-1) ${ }^{-/-}$MEF, dendritic cells (DC), macrophages, and neurons sustained higher WNV titers and failed to induce a type I IFN response [41, 42]. While $\mathrm{MaVs}^{-1-}$ mice showed enhanced spread and replication in peripheral tissues, and uniform lethality with virulent WNV strains [42], RLR alone do not exclusively detect WNV as $\mathrm{MaVs}^{-/-}$mice produced higher levels of systemic type I IFN than wild type controls.

\section{Protein kinase R (PKR)}

The dsRNA-dependent protein kinase R (PKR) is both a cytosolic PRR that detects dsRNA and an ISG with effector functions capable of controlling viral replication by blocking translation of viral and cellular mRNA. PKR can serve as a PRR by binding dsRNA, which promotes its activation and signaling actions. PKR can regulate antiviral responses by suppressing mRNA translation through phosphorylation of the alpha subunit of eukaryotic initiation factor 2 (eIF2) and by modulating IFN mRNA stability [43]. PKR has been implicated in modulating IFN signaling during WNV infection [44]. While some studies suggest that virulent WNV infection does not directly activate PKR [45], in cells infected with attenuated, chimeric WNV strains viral RNA can serve as ligands for PKR as it is not completely protected by intracellular membranes [46]. The specific role of PKR in vivo was inferred from studies with Rnase $^{-1-}$ and Eif2ak2(PKR) ${ }^{-/-}$x Rnase ${ }^{-1-}$ mice: more severe virological and survival phenotypes were observed in Eif2ak2 $2^{-/}$x Rnase $^{-/-}$mice infected with WNV [47]. Eif2ak2 $2^{-1-}$ mice showed no defect in type I IFN response in serum suggesting a limited contribution of PKR in systemic IFN induction. Rather, PKR appeared to contribute to antiviral effector functions, as IFN- $\beta$ pretreated Eif2ak2 $2^{-1}$ macrophages and cortical neurons were more susceptible to WNV infection relative to wild type cells.

\section{Endosomal Toll-like receptors}

Recognition of WNV by TLRs occurs in endosomes and is largely mediated by TLR7 and TLR3, which bind ssRNA and dsRNA, respectively. TLR7 recruits the adaptor protein MyD88 and forms a complex with TRAF3, TRAF6, IRAK1 and IRAK4. This complex recruits TAK1, a kinase that activates NF- $\kappa$ B, or TBK1 and $\mathrm{\kappa} \kappa-\varepsilon$ kinases, which in turn stimulate IRF-3 and/or IRF-7 and IFN responses. TLR3, in contrast, uses TRIF as an adaptor protein. TRIF can stimulate an IRF-3 and IRF-7-dependent induction of type I IFN genes via its interaction with TRAF3, TBK1 and I $\kappa-\varepsilon$ (Figure 1).

Despite several studies showing that ligation of TLR3 by dsRNA in vitro regulates IFN and other cytokine responses, its role in inducing IFN and protecting against viral infection in vivo remains less clear (reviewed in [48]). For WNV, two studies using the same TIr $3^{-1-}$ mice reported distinct phenotypes: One study showed a detrimental role, as $T 1 r 3^{-1-}$ mice had improved survival rates after WNV infection; Tlr $3^{-1-}$ mice showed a mildly increased WNV burden in peripheral tissues with a decreased systemic pro-inflammatory cytokine response that was associated with reduced blood-brain barrier permeability and entry of WNV into the brain [49]. A second study showed a protective role with decreased survival of $T / 3^{-/-}$ mice after WNV infection, mildly elevated viral titers in peripheral tissues, and early viral spread to the CNS [50]. Ex vivo studies showed a dispensable role of TLR3 in regulating IFN responses and controlling WNV replication in MEF, DC, and macrophages. TLR3 may have a more significant role in the CNS, potentially by restricting WNV replication in neurons [50] or via dsRNA sensing and induction of pro-inflammatory cytokines in resident cells of the CNS, including microglia and astrocytes [51, 52]. WNV NS1 protein has been reported to inhibit TLR3 signal transduction by attenuating transcriptional activation of the IFN- $\beta$ and NF- $\kappa$ B promoters $[53,54]$, although this finding was not observed in a second 
study [55]. The exact contribution of TLR3 in WNV protection and/or pathogenesis requires further study but likely involves both cell-intrinsic and -extrinsic effects in the CNS.

The function of TLR7 in WNV infection in vivo has been examined in two studies using the same $T I r 7^{-1}$ mice, and again, somewhat discordant phenotypes were described [56, 57]. One study found no difference in susceptibility to WNV between wild type and $T I r 7^{-1-}$ mice after intradermal injection or feeding by infected mosquitoes. Viral load analysis revealed similar levels of WNV RNA in the peripheral tissues and brains of these two groups of mice [56, 57]. In the second study, $T / r 7^{-1-}$ mice were more vulnerable to intraperitoneal WNV infection and showed increased viremia and defects in immune cell homing to WNVinfected tissues via an IL-23-dependent mechanism [56]. Both studies reported increased systemic levels of pro-inflammatory cytokines (IL-6, TNF-a, and IL-12) and type I IFN in $T I r 7^{-1-}$ mice when compared to wild type animals, which could be due to differential replication in key cell types. Thus, abrogation of the TLR7 signaling pathway, which eliminates type I IFN production in pDC, had little systemic impact on IFN production after WNV infection. Consistent with this, recent studies with diphtheria toxin receptor transgenic mice that selectively deplete pDCs showed only small decreases in type I IFN production after viral infection [17].

Two studies reported a protective effect of MyD88 [56, 58], the downstream adaptor molecule for several TLR, including TLR7. $M y d 88^{-/-}$mice showed elevated viral burden primarily in the brain, even though little effect on the systemic type I IFN response was observed. In cell culture, increased WNV replication was observed in $M y d 88^{-1-}$ macrophages and subsets of neurons. An absence of MyD88 had an independent and negative effect on recruitment of monocyte-derived macrophages and $\mathrm{T}$ cells into the brain; this was associated with blunted induction of the chemokines that attract leukocytes, which function to clear WNV infection from the CNS. Together, these experiments suggest that MyD88 restricts WNV by inhibiting replication and modulating immune cell migration into the CNS.

\section{Transcriptional regulation of the type I IFN response after WNV infection}

The IRF family is composed of nine related members that function as transcription factors [59]. IRF-3 and IRF-7 have been identified as the key transcriptional regulators of type I IFN genes following viral infection, whereas IRF-1 and IRF-5 are thought to contribute subordinately to this response. Optimal activation of the type I IFN response after viral infection is believed to occur following a two-step amplification sequence. Viral sensing by PRR (TLR and RLR) triggers nuclear translocation of constitutively expressed IRF-3 with subsequent production of IFN- $\beta$ and IFN- $\alpha 4$ by the infected cell. The newly synthesized IFN binds to IFNAR, activates JAK-STAT signaling, and induces expression of hundreds of ISGs, including IRF-7. Subsequent activation of IRF-7 amplifies expression of IFN- $\beta$ and many other IFN-a subtypes through a positive feedback loop. Small amounts of constitutively expressed IRF-7 in some cell types, however, may prime the type I IFN response directly [60]. Recent studies with WNV have helped to elucidate the role of individual IRF family members in regulating the type IFN response.

\section{In vivo studies}

Infection of $I r f 3^{--}$or $I r f 7^{-1-}$ mice with WNV resulted in severe pathology linked with a lack of innate antiviral immunity [22, 61]. Consistent with this, polymorphisms in the IRF-3 gene associate with symptomatic WNV infection in humans [62]. In both $I r f 3^{-1-}$ and Irf $7^{-1-}$ mice, enhanced viral burden was observed in peripheral tissues (spleen, kidney, blood, and lymph node), which led to entry and sustained replication in the CNS. Analysis of serum from Irf3 ${ }^{-1-}$ mice infected with WNV or WNV-virus-like-particles established a dispensable 
role of IRF-3 in regulating systemic production of type I IFN [22, 63]. In contrast, WNVinfected $I f f 7^{-1}$ mice showed blunted type I IFN responses [61]. The IRF-7-dependent production of IFN in blood appears independent of TLR7, as levels were not decreased in $T I r 7^{-1-}$ mice after WNV infection [56]. Thus, it remains uncertain as to which cell type regulates systemic type I IFN production after WNV infection.

Recent studies have investigated the antiviral role of IRF-1 in WNV infection, as it was originally described as a regulator of type I IFN responses after virus infection [64]. Irf $1^{-1-}$ mice were vulnerable to lethal WNV infection with enhanced viral replication in peripheral tissues and rapid dissemination into the CNS [65]. IRF-1, in contrast to IRF-3 and IRF-7, also had an independent effect on $\mathrm{CD}^{+} \mathrm{T}$ cell expansion. Although markedly fewer $\mathrm{CD} 8^{+} \mathrm{T}$ cells are observed in naïve animals, Irf $1^{-1-}$ mice rapidly expanded their pool of WNVspecific cytolytic $\mathrm{CD}^{+} \mathrm{T}$ cells. Thus, IRF- 1 restricts WNV infection by modulating the expression of innate antiviral effector molecules while shaping the antigen-specific $\mathrm{CD} 8^{+} \mathrm{T}$ cell response.

\section{Cell-specific innate defenses}

Macrophages are susceptible to WNV infection [15] and are more permissive in the absence of IFN signaling [1]. In macrophages, IRF-3 was identified as an essential regulator of the basal expression of host defense molecules including IFIT-1, IFIT-2, RIG-I and MDA5, potentially controlling the permissiveness of this cell type for WNV. The contribution of IRF-3 and IRF-7 in macrophages was more fully established in subsequent studies with Irf $7^{-1-}$ or $\operatorname{Irf3^{-1-}}$ x Irf $7^{-1-}$ DKO cells. While a deficiency of IRF-7 completely abrogated the IFN-a response, no effect on IFN- $\beta$ gene induction was observed in $\mathrm{Irf}^{-1-}$ cells. Analysis of the IFN- $\beta$ response in $I r f 3^{-1-}$ x Irf $7^{-1-}$ DKO macrophages revealed an unexpected finding: this response was completely abolished at early time points after WNV infection but was not substantially altered at late time points [41]. Thus, IRF-3 and IRF-7 only partially regulate the IFN- $\beta$ gene and ISG expression in macrophages. Consistent with this, Irf $1^{-1-}$ macrophages supported enhanced WNV replication and showed diminished cell-intrinsic innate immune responses, and ectopic expression of IRF-1 inhibited WNV infection in transformed cells [65, 66]. Moreover, IRF-1 gene expression in WNV-infected cells can be induced through an IRF-3 -and IRF-7-independent pathway [67]. Thus, at least for some macrophage subsets, the type I IFN response appears to be mediated through the combination of IRF-1, IRF-3, and IRF-7 function.

DCs have primary roles in regulating innate and adaptive immune responses against viruses, and also are targets for WNV infection in vivo [57, 68]. Although pDC potently produce type I IFN after WNV infection [20], other DC subsets are major sources of type I IFN in response to viral infection [69]. Indeed, myeloid DC, when productively infected with WNV, secrete large amounts of type I IFN [20, 70], and impaired IFN signaling in DCs is observed in older human donors infected with WNV [23]. The IRF-3 and IRF-7-dependent regulation of type I IFN gene induction in $\mathrm{mDC}$ differs compared to that seen with macrophages. Whereas the IFN- $\alpha$ response was analogously regulated by IRF-7, the IFN- $\beta$ response in mDCs was largely unaffected by the absence of IRF-1, IRF-3, IRF-7, or a combination of IRF-3 and IRF-7 [41] (Figure 2). These findings may reflect an undocumented yet important role of IRF-5 or other transcription factors in transmitting an IPS-1-dependent signal downstream of RLR recognition of viral RNA to induce IFN- $\beta$ expression in specific cell types such as DCs.

\section{IFN stimulated genes that control WNV infection}

Progress has been made recently in defining the specific ISGs that limit WNV infection. Systematic investigation of the antiviral functions of large groups of ISGs using ectopic 
gene or shRNA screens $[66,71]$ has identified new genes and gene families that restrict infection of flaviviruses, including WNV (Table 1).

Initial studies showed that $2^{\prime}-5^{\prime}$-oligoadenylate synthase (Oas) proteins mediate intrinsic cell resistance to WNV. Because RNase L is activated by $2^{\prime}-5^{\prime}$-linked oligoadenylates that are synthesized by Oas enzymes, several groups have assessed its antiviral function in the context of WNV infection. RNAse L inhibits viral infections by cleaving viral RNA [72] and by generating small self-RNA that amplify immune responses through an RLRdependent pathway [73]. Rnase $^{-1}$ macrophages and MEFs supported increased WNV replication $[47,74]$ and a deficiency of RNAse L partially rescued a WNV mutant deficient in the production of a non-coding subgenomic flavivirus RNA (sfRNA) [6]. Mice deficient in RNase L showed moderately increased lethality following WNV infection, with higher viral loads in peripheral tissues at early time points [47].

Although susceptibility to flaviviruses in mice has been mapped to a mutation in Oas $1 b$, resulting in the expression of a truncated protein $[75,76]$, the antiviral mechanism by this gene remains unknown but appears independent of RNAse L [74, 77]. Genetic variation in $O A S 1$ also has been suggested as a risk factor for initial infection with WNV in humans [78]. Knock-in of the wild type Oas $1 b$ allele into a flavivirus-induced disease susceptible mouse generated a resistant phenotype [79], and murine cells that ectopically express wild type Oas $1 b$ resisted WNV infection by preventing viral RNA accumulation [80]. While biochemical studies have shown that Oas $1 \mathrm{~b}$ itself is an inactive $2^{\prime}-5^{\prime}$ Oas, recent experiments suggest that Oas $1 \mathrm{~b}$ inhibits Oas $1 \mathrm{a}$ activity resulting in reduced $2^{\prime}-5^{\prime}$ oligoA production [77]. Negative regulation of $2^{\prime}-5^{\prime}$ Oas by inactive Oas $1 \mathrm{~b}$ proteins may fine-tune the RNase $\mathrm{L}$ response that could cause significant damage in cells, if it were not tightly controlled.

The ISG viperin ( $r s a d 2)$ can inhibit WNV infection in cells and in vivo. Viperin is induced in many cell types after exposure to type I and II IFN, TLR agonists, or virus infection [81]. Viperin localizes to the cytosolic face of the endoplasmic reticulum via an amphipathic ahelix [82], where it is believed to inhibit infection of several viruses by modulating cholesterol and isoprenoid biosynthesis, lipid raft formation, and the composition and localization of lipid droplets. Viperin originally was identified as a possible inhibitor of WNV infection in ectopic expression studies in cell culture [71]. Subsequent in vivo studies confirmed its antiviral potential, as $\mathrm{Rsad}^{-/-}$mice infected with WNV showed increased lethality and enhanced viral replication in CNS tissues [83].

Members of the IFIT family of genes (Ifit1, Ifit2, and Ifit3 in mice) also likely contribute to the control of WNV infection. Initial studies suggested that IFIT proteins exerted their antiviral function by inhibiting protein translation through interaction with subunits of translation initiation factor eIF3 (reviewed in [84]). In cell culture and mouse models of infection, WNV strongly induces Ifit 1 gene expression in target cells via IFN-dependent and -independent signaling pathways $[22,85]$. Interestingly, a WNV mutant with a site-specific substitution in the NS5 gene (WNV-E218A) that abolishes $2^{\prime}-O$-methyltransferase activity [86] showed wild-type levels of replication in transformed Vero and BHK21 cells but attenuated infection in primary macrophages and wild type mice [3]. Because replication of WNV-E218A was rescued in the absence of IFNAR or Ifit1 [3], 2' $-O$ methylation of the $5^{\prime}$ cap of WNV RNA functions to subvert host antiviral responses through escape of Ifit1mediated suppression. Differential methylation of cytoplasmic RNA probably serves as an example for pattern recognition and restriction of propagation of some foreign viral RNA in host cells. 
Additional ISGs have been suggested to inhibit WNV infection based on studies in cell culture. IFITM3 was recently shown to inhibit an early entry step in infection of WNV in cells [87], an observation that was confirmed by ectopic expression studies in HEK293 and STAT $1^{-1}$ cells $[66,71]$. ISG20 also has been proposed to inhibit infection of flaviviruses, including WNV [71, 88]. Newer genetic screens have identified additional candidate ISGs (C6orf150 (MB21D1), HPSE, and NAMPT) with anti-WNV activity [66, 71]. Although the field is rapidly advancing with respect to identifying putative antiviral ISG against WNV and other flaviviruses, future studies in genetically deficient animals will be needed to establish the cell- and tissue-specific non-redundant effects and hierarchy in controlling infection in the context of a type I IFN response.

\section{Concluding remarks}

The innate immune response of the host is programmed to control viral replication and limit spread by recognizing non-self nucleic acid as PAMPs and triggering an antiviral response. Although type I IFN was discovered more than 50 years ago, only recently have the mechanisms by which these molecules are induced, signal, and produce an antiviral effect been delineated. The generation and distribution of mice with targeted deletions in PRR, transcription factors, IFN signaling or effector genes has allowed the field to dissect and evaluate the function of individual host defense genes in the context of infection by specific viruses. WNV is a zoonotic pathogen that readily infects mice, and thus has afforded a unique perspective on the interface between the mammalian cell-intrinsic innate host response and viral pathogenesis. In the last few years, the field has identified the specific PRR and signaling pathways that detect entry and infection by WNV and initiate a protective IFN response. Over the next five years, we anticipate that several important questions will be answered. Among these include: (a) the identification of the specific PAMPs on WNV that are recognized by PRR; (b) the characterization of a hierarchy of ISGs that function to control different stages of WNV infection in distinct cell types and tissues; (c) the role of viral proteins, viral RNA structural elements, and viral non-coding RNA in modulating cell-intrinsic innate immune responses to WNV; and (d) the basis for WNV virulence, and how it interfaces with recognition or evasion of cell-intrinsic immune pathways. As these topics are explored, the field undoubtedly will gain new insight into fundamental cellular responses as well as mechanisms of viral pathogenesis. This work may promote novel strategies for development of therapeutic agents that activate specific innate immune processes or effector molecules to contain spread and disease of WNV, and likely many other viruses.

\section{Acknowledgments}

The authors would like to acknowledge support from the National Institutes of Health for support of work in their laboratories (U54 AI081680 and U54 AI057160 (Pacific Northwest and Midwest Regional Center of Excellence for Biodefense and Emerging Infectious Diseases Research), U19 AI083019, and R01 AI074973.

\section{REFERENCES}

1. Samuel MA, Diamond MS. Type I IFN protects against lethal West Nile Virus infection by restricting cellular tropism and enhancing neuronal survival. J Virol. 2005; 79:13350-13361. [PubMed: 16227257]

2. Keller BC, et al. Resistance to alpha/beta interferon is a determinant of West Nile virus replication fitness and virulence. J Virol. 2006; 80:9424-9434. [PubMed: 16973548]

3. Daffis $\mathrm{S}$, et al. $2^{\prime}-\mathrm{O}$ methylation of the viral mRNA cap evades host restriction by IFIT family members. Nature. 2010; 468:452-456. [PubMed: 21085181]

4. Daffis $S$, et al. The naturally attenuated Kunjin strain of West Nile virus shows enhanced sensitivity to the host type I interferon response. J Virol. 2011; 85:5664-5668. [PubMed: 21411525] 
5. Liu WJ, et al. A single amino acid substitution in the West Nile virus nonstructural protein NS2A disables its ability to inhibit alpha/beta interferon induction and attenuates virus virulence in mice. $\mathrm{J}$ Virol. 2006; 80:2396-2404. [PubMed: 16474146]

6. Schuessler A, et al. West Nile virus non-coding subgenomic RNA contributes to viral evasion of type I interferon-mediated antiviral response. J Virol. 2012

7. Winkelmann ER, et al. Intrinsic adjuvanting of a novel single-cycle flavivirus vaccine in the absence of type I interferon receptor signaling. Vaccine. 2012; 30:1465-1475. [PubMed: 22226862]

8. Liu WJ, et al. Inhibition of interferon signaling by the New York 99 strain and kunjin subtype of West Nile virus involves blockage of STAT1 and STAT2 activation by nonstructural proteins. J Virol. 2005; 79:1934-1942. [PubMed: 15650219]

9. Perwitasari O, et al. Inhibitor of kappaB Kinase \{epsilon\} (IKK\{epsilon\}), STAT1, and IFIT2 Proteins Define Novel Innate Immune Effector Pathway against West Nile Virus Infection. J Biol Chem. 2011; 286:44412-44423. [PubMed: 22065572]

10. Ben-Nathan D, et al. West Nile virus neuroinvasion and encephalitis induced by macrophage depletion in mice. Arch Virol. 1996; 141:459-469. [PubMed: 8645088]

11. Purtha WE, et al. Early B-cell activation after West Nile virus infection requires alpha/beta interferon but not antigen receptor signaling. J Virol. 2008; 82:10964-10974. [PubMed: 18786989]

12. Lin YL, et al. Inhibition of Japanese encephalitis virus infection by nitric oxide: antiviral effect of nitric oxide on RNA virus replication. J Virol. 1997; 71:5227-5235. [PubMed: 9188590]

13. Saxena SK, et al. Antiviral effect of nitric oxide during Japanese encephalitis virus infection. Int $\mathbf{J}$ Exp Pathol. 2000; 81:165-172. [PubMed: 10762444]

14. Shrestha B, et al. Tumor necrosis factor alpha protects against lethal West Nile virus infection by promoting trafficking of mononuclear leukocytes into the central nervous system. J Virol. 2008; 82:8956-8964. [PubMed: 18632856]

15. Kong KF, et al. West nile virus attenuates activation of primary human macrophages. Viral Immunol. 2008; 21:78-82. [PubMed: 18355125]

16. Rios M, et al. Monocytes-macrophages are a potential target in human infection with West Nile virus through blood transfusion. Transfusion. 2006; 46:659-667. [PubMed: 16584445]

17. Swiecki M, et al. Plasmacytoid dendritic cell ablation impacts early interferon responses and antiviral NK and CD8(+) T cell accrual. Immunity. 2010; 33:955-966. [PubMed: 21130004]

18. Sun $\mathrm{P}$, et al. Functional characterization of ex vivo blood myeloid and plasmacytoid dendritic cells after infection with dengue virus. Virology. 2009; 383:207-215. [PubMed: 19013627]

19. Wang JP, et al. Flavivirus activation of plasmacytoid dendritic cells delineates key elements of TLR7 signaling beyond endosomal recognition. J Immunol. 2006; 177:7114-7121. [PubMed: 17082628]

20. Silva MC, et al. Differential activation of human monocyte-derived and plasmacytoid dendritic cells by West Nile virus generated in different host cells. J Virol. 2007; 81:13640-13648. [PubMed: 17913823]

21. Steinbrink K, et al. Myeloid dendritic cell: From sentinel of immunity to key player of peripheral tolerance? Hum Immunol. 2009; 70:289-293. [PubMed: 19217922]

22. Daffis $S$, et al. Cell-specific IRF-3 responses protect against West Nile virus infection by interferon-dependent and independent mechanisms. PLoS Pathog. 2007; 3:e106. [PubMed: 17676997]

23. Qian F, et al. Impaired interferon signaling in dendritic cells from older donors infected in vitro with West Nile virus. J Infect Dis. 2011; 203:1415-1424. [PubMed: 21398396]

24. Hildner K, et al. Batf3 deficiency reveals a critical role for CD8alpha+ dendritic cells in cytotoxic T cell immunity. Science. 2008; 322:1097-1100. [PubMed: 19008445]

25. Davison AM, King NJ. Accelerated dendritic cell differentiation from migrating Ly6C(lo) bone marrow monocytes in early dermal West Nile virus infection. J Immunol. 2011; 186:2382-2396. [PubMed: 21248254]

26. Loo YM, Gale M Jr. Immune signaling by RIG-I-like receptors. Immunity. 2011; 34:680-692. [PubMed: 21616437] 
27. Schlee M, et al. Approaching the RNA ligand for RIG-I? Immunol Rev. 2009; 227:66-74. [PubMed: 19120476]

28. Yoneyama M, Fujita T. Structural mechanism of RNA recognition by the RIG-I-like receptors. Immunity. 2008; 29:178-181. [PubMed: 18701081]

29. Berke IC, Modis Y. MDA5 cooperatively forms dimers and ATP-sensitive filaments upon binding double-stranded RNA. Embo J. 2012; 31:1714-1726. [PubMed: 22314235]

30. Gack MU, et al. TRIM25 RING-finger E3 ubiquitin ligase is essential for RIG-I-mediated antiviral activity. Nature. 2007; 446:916-920. [PubMed: 17392790]

31. Gack MU, et al. Roles of RIG-I N-terminal tandem CARD and splice variant in TRIM25-mediated antiviral signal transduction. Proc Natl Acad Sci U S A. 2008; 105:16743-16748. [PubMed: 18948594]

32. Hou F, et al. MAVS forms functional prion-like aggregates to activate and propagate antiviral innate immune response. Cell. 2011; 146:448-461. [PubMed: 21782231]

33. Horner SM, et al. Mitochondrial-associated endoplasmic reticulum membranes (MAM) form innate immune synapses and are targeted by hepatitis C virus. Proc Natl Acad Sci U S A. 2011; 108:14590-14595. [PubMed: 21844353]

34. Dixit E, et al. Peroxisomes are signaling platforms for antiviral innate immunity. Cell. 2010; 141:668-681. [PubMed: 20451243]

35. Wang P, et al. Caspase-12 controls West Nile virus infection via the viral RNA receptor RIG-I. Nat Immunol. 2010; 11:912-919. [PubMed: 20818395]

36. Liu HM, et al. The mitochondrial targeting chaperone $14-3-3 \varepsilon$ regulates a RIG-I translocon that mediates membrane association and innate antiviral immunity. Cell Host and Microbe. 2012; 11:1-10. [PubMed: 22264506]

37. Loo YM, et al. Distinct RIG-I and MDA5 signaling by RNA viruses in innate immunity. J Virol. 2008; 82:335-345. [PubMed: 17942531]

38. Fredericksen BL, Gale M Jr. West Nile virus evades activation of interferon regulatory factor 3 through RIG-I-dependent and -independent pathways without antagonizing host defense signaling. J Virol. 2006; 80:2913-2923. [PubMed: 16501100]

39. Fredericksen BL, et al. Establishment and maintenance of the innate antiviral response to West Nile virus involves both RIG-I and MDA5 signaling through IPS-1. J Virol. 2008; 82:609-616. [PubMed: 17977974]

40. Kato H, et al. Differential roles of MDA5 and RIG-I helicases in the recognition of RNA viruses. Nature. 2006; 441:101-105. [PubMed: 16625202]

41. Daffis $\mathrm{S}$, et al. Induction of IFN-beta and the innate antiviral response in myeloid cells occurs through an IPS-1-dependent signal that does not require IRF-3 and IRF-7. PLoS Pathog. 2009; 5:e1000607. [PubMed: 19798431]

42. Suthar MS, et al. IPS-1 is essential for the control of West Nile virus infection and immunity. PLoS Pathog. 2010; 6:e1000757. [PubMed: 20140199]

43. Schulz $\mathrm{O}$, et al. Protein kinase $\mathrm{R}$ contributes to immunity against specific viruses by regulating interferon mRNA integrity. Cell Host Microbe. 2010; 7:354-361. [PubMed: 20478537]

44. Gilfoy FD, Mason PW. West Nile virus-induced IFN production is mediated by the doublestranded RNA-dependent protein kinase, PKR. J Virol. 2007; 81:11148-11158. [PubMed: 17686861]

45. Elbahesh $\mathrm{H}$, et al. West Nile virus infection does not induce PKR activation in rodent cells. Virology. 2011; 421:51-60. [PubMed: 21982595]

46. Courtney SC, et al. West nile virus infections suppress early viral RNA synthesis and avoid inducing the cell stress granule response. J Virol. 2012; 86:3647-3657. [PubMed: 22258263]

47. Samuel MA, et al. PKR and RNAse L contribute to protection against lethal West Nile virus infection by controlling early viral spread in the periphery and replication in neurons. J Virol. 2006; 80:7009-7019. [PubMed: 16809306]

48. Vercammen E, et al. Sensing of viral infection and activation of innate immunity by toll-like receptor 3. Clin Microbiol Rev. 2008; 21:13-25. [PubMed: 18202435] 
49. Wang T, et al. Toll-like receptor 3 mediates West Nile virus entry into the brain causing lethal encephalitis. Nat Med. 2004; 10:1366-1373. [PubMed: 15558055]

50. Daffis S, et al. Toll-like receptor 3 has a protective role against West Nile virus infection. J Virol. 2008; 82:10349-10358. [PubMed: 18715906]

51. Town T, et al. Microglia recognize double-stranded RNA via TLR3. J Immunol. 2006; 176:38043812. [PubMed: 16517751]

52. Kim H, et al. Double-stranded RNA mediates interferon regulatory factor 3 activation and interleukin- 6 production by engaging Toll-like receptor 3 in human brain astrocytes. Immunology. 2008

53. Scholle F, Mason PW. West Nile virus replication interferes with both poly(I:C)-induced interferon gene transcription and response to interferon treatment. Virology. 2005; 342:77-87. [PubMed: 16111732]

54. Wilson JR, et al. West Nile virus nonstructural protein 1 inhibits TLR3 signal transduction. J Virol. 2008; 82:8262-8271. [PubMed: 18562533]

55. Baronti C, et al. Nonstructural NS1 proteins of several mosquito-borne Flavivirus do not inhibit TLR3 signaling. Virology. 2010; 404:319-330. [PubMed: 20554300]

56. Town T, et al. Toll-like receptor 7 mitigates lethal West Nile encephalitis via interleukin 23dependent immune cell infiltration and homing. Immunity. 2009; 30:242-253. [PubMed: 19200759]

57. Welte T, et al. Toll-like receptor 7-induced immune response to cutaneous West Nile virus infection. J Gen Virol. 2009; 90:2660-2668. [PubMed: 19641044]

58. Szretter KJ, et al. The innate immune adaptor molecule MyD88 restricts West Nile replication and spread in neurons of the central nervous system. J Virol. 2010; 84:12125-12138. [PubMed: 20881045]

59. Tamura T, et al. The IRF family transcription factors in immunity and oncogenesis. Annu Rev Immunol. 2008; 26:535-584. [PubMed: 18303999]

60. Honda K, Taniguchi T. IRFs: master regulators of signalling by Toll-like receptors and cytosolic pattern-recognition receptors. Nat Rev Immunol. 2006; 6:644-658. [PubMed: 16932750]

61. Daffis S, et al. Interferon regulatory factor IRF-7 induces the antiviral alpha interferon response and protects against lethal West Nile virus infection. J Virol. 2008; 82:8465-8475. [PubMed: 18562536]

62. Bigham AW, et al. Host genetic risk factors for West Nile virus infection and disease progression. PloS one. 2011; 6:e24745. [PubMed: 21935451]

63. Bourne N, et al. Early production of type I interferon during West Nile virus infection: role for lymphoid tissues in IRF3-independent interferon production. J Virol. 2007; 81:9100-9108. [PubMed: 17567689]

64. Fujita T, et al. Induction of endogenous IFN-alpha and IFN-beta genes by a regulatory transcription factor, IRF-1. Nature. 1989; 337:270-272. [PubMed: 2911367]

65. Brien JD, et al. Interferon Regulatory Factor-1 (IRF-1) Shapes Both Innate and CD8 T Cell Immune Responses against West Nile Virus Infection. PLoS Pathog. 2011; 7:e1002230. [PubMed: 21909274]

66. Schoggins JW, et al. A diverse range of gene products are effectors of the type I interferon antiviral response. Nature. 2011; 472:481-485. [PubMed: 21478870]

67. Pulit-Penaloza JA, et al. Type 1 IFN-independent activation of a subset of interferon stimulated genes in West Nile virus Eg101-infected mouse cells. Virology. 2012; 425:82-94. [PubMed: 22305622]

68. Martina BE, et al. DC-SIGN enhances infection of cells with glycosylated West Nile virusin vitro and virus replication in human dendritic cells induces production of IFN-alpha and TNF-alpha. Virus Res. 2008; 135:64-71. [PubMed: 18405996]

69. Fitzgerald-Bocarsly P, Feng D. The role of type I interferon production by dendritic cells in host defense. Biochimie. 2007; 89:843-855. [PubMed: 17544561]

70. Daffis S, et al. Measure and countermeasure: type I IFN (IFN-alpha/beta) antiviral response against West Nile virus. Journal of innate immunity. 2009; 1:435-445. [PubMed: 20375601] 
71. Jiang D, et al. Identification of five interferon-induced cellular proteins that inhibit west nile virus and dengue virus infections. J Virol. 2010; 84:8332-8341. [PubMed: 20534863]

72. Zhou A, et al. Interferon action and apoptosis are defective in mice devoid of $2^{\prime}, 5^{\prime}$-oligoadenylatedependent RNase L. Embo J. 1997; 16:6355-6363. [PubMed: 9351818]

73. Malathi K, et al. Small self-RNA generated by RNase L amplifies antiviral innate immunity. Nature. 2007; 448:816-819. [PubMed: 17653195]

74. Scherbik SV, et al. RNase L plays a role in the antiviral response to West Nile virus. J Virol. 2006; 80:2987-2999. [PubMed: 16501108]

75. Perelygin AA, et al. Positional cloning of the murine flavivirus resistance gene. Proc Natl Acad Sci U S A. 2002; 99:9322-9327. [PubMed: 12080145]

76. Mashimo T, et al. A nonsense mutation in the gene encoding $2^{\prime}-5^{\prime}$-oligoadenylate synthetase/L1 isoform is associated with West Nile virus susceptibility in laboratory mice. Proc Natl Acad Sci U S A. 2002; 99:11311-11316. [PubMed: 12186974]

77. Elbahesh H, et al. The Flvr-encoded murine oligoadenylate synthetase 1b (Oas1b) suppresses 2-5A synthesis in intact cells. Virology. 2011; 409:262-270. [PubMed: 21056894]

78. Lim JK, et al. Genetic variation in OAS1 is a risk factor for initial infection with West Nile virus in man. PLoS Pathog. 2009; 5:e1000321. [PubMed: 19247438]

79. Scherbik SV, et al. Knock-in of the Oas1b(r) allele into a flavivirus-induced disease susceptible mouse generates the resistant phenotype. Virology. 2007; 368:232-237. [PubMed: 17904183]

80. Kajaste-Rudnitski A, et al. The $2^{\prime}, 5^{\prime}$-oligoadenylate synthetase $1 \mathrm{~b}$ is a potent inhibitor of West Nile virus replication inside infected cells. J Biol Chem. 2006; 281:4624-4637. [PubMed: 16371364]

81. Fitzgerald KA. The interferon inducible gene: Viperin. J Interferon Cytokine Res. 2011; 31:131135. [PubMed: 21142818]

82. Hinson ER, Cresswell P. The antiviral protein, viperin, localizes to lipid droplets via its N-terminal amphipathic alpha-helix. Proc Natl Acad Sci U S A. 2009; 106:20452-20457. [PubMed: 19920176]

83. Szretter KJ, et al. The interferon-inducible gene viperin restricts West Nile virus pathogenesis. J Virol. 2011; 85:11557-11566. [PubMed: 21880757]

84. Fensterl V, Sen GC. The ISG56/IFIT1 Gene Family. J Interferon Cytokine Res. 2011; 31:71-78. [PubMed: 20950130]

85. Wacher C, et al. Coordinated regulation and widespread cellular expression of interferonstimulated genes (ISG) ISG-49, ISG-54, and ISG-56 in the central nervous system after infection with distinct viruses. J Virol. 2007; 81:860-871. [PubMed: 17079283]

86. Zhou Y, et al. Structure and function of flavivirus NS5 methyltransferase. J Virol. 2007; 81:38913903. [PubMed: 17267492]

87. Brass AL, et al. The IFITM proteins mediate cellular resistance to influenza A H1N1 virus, West Nile virus, and dengue virus. Cell. 2009; 139:1243-1254. [PubMed: 20064371]

88. Zhou Z, et al. Antiviral activities of ISG20 in positive-strand RNA virus infections. Virology. 2011; 409:175-188. [PubMed: 21036379]

89. Szretter KJ, et al. $2^{\prime}$-O methylation of the viral mRNA cap by West Nile virus evades Ifit1dependent and -independent mechanisms of host restriction in vivo. PLoS Pathog. 2012 In press.

90. Chan YK, et al. IFITM Proteins Restrict Antibody-Dependent Enhancement of Dengue Virus Infection. PloS one. 2012; 7:e34508. [PubMed: 22479637]

91. Taylor RT, et al. TRIM79alpha, an interferon-stimulated gene product, restricts tick-borne encephalitis virus replication by degrading the viral RNA polymerase. Cell Host Microbe. 2011; 10:185-196. [PubMed: 21925107]

92. Evans JD, et al. West Nile virus infection induces depletion of IFNAR1 protein levels. Viral Immunol. 2011; 24:253-263. [PubMed: 21830897]

93. Guo JT, et al. West Nile virus inhibits the signal transduction pathway of alpha interferon. J Virol. 2005; 79:1343-1350. [PubMed: 15650160]

94. Mackenzie JM, et al. Cholesterol manipulation by West Nile virus perturbs the cellular immune response. Cell Host Microbe. 2007; 2:229-239. [PubMed: 18005741] 


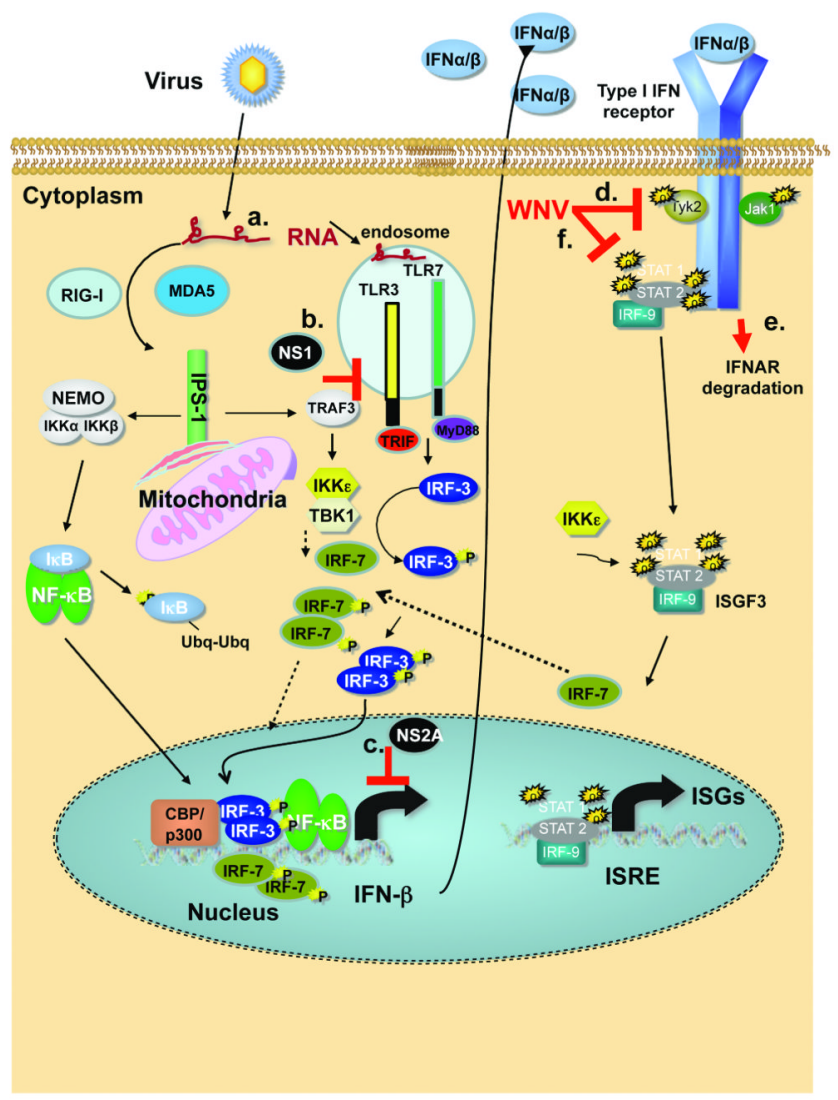

Figure 1. Detection of WNV and activation of IFN- $\alpha / \beta$ genes and ISG

Schematic of innate immune signaling triggered by WNV infection through cytosolic RLRs or endosomal TLRs. Solid lines indicate the primary response. Broken lines indicate the amplification response driven by IRF-7 after initial IFN production by the infected cell. P denotes phosphorylation; Ubq denotes ubiquitin modification. Infection by WNV produces RNA intermediates in the cytosol that are detected as non-self by the host RLR. RIG-I acts as the main sensor for WNV during the early steps of infection. RIG-I activation promotes interaction with IPS-1 that leads to the recruitment of TRAF3, TBK1 and I $\kappa \kappa-\varepsilon$ or NEMO, I $\kappa \kappa-\alpha$, and I $\kappa \kappa-\beta$, which results in activation and nuclear translocation of IRF-3 and NF$\kappa B$, respectively. Small amounts of constitutively produced IRF-7 also may be activated via this pathway. IRF-3, IRF-7, and NF- $\kappa$ B bind the IFN- $\beta$ gene promoter and induce transcription. Secretion of IFN- $\beta$ by the infected cells results in paracrine type I IFN signaling through IFNAR. Activation of IFNAR induces phosphorylation of JAK1 and Tyk2, which can promote the formation of the heterotrimer ISGF3 formed by STAT1, STAT2 and IRF-9. Ultimately, translocation into the nucleus of ISGF3 induces hundreds of interferon-stimulated genes (ISG), including IRF-7. During late phases of infection, detection of WNV also relies on MDA5, whereas I $\kappa \kappa-\varepsilon$ promotes specific ISG induction through phosphorylation of STAT1 on serine 708. Induction of IFN-a genes (except IFNa4) occurs mainly via the transcriptional activity of IRF-7. Putative mechanisms by which WNV antagonizes the type I IFN response are marked and include (a) a delay in recognition of WNV RNA by RIG-I [38]; (b) attenuation of TLR3 signaling by the NS1 protein [54]; (c) reduction in IFN- $\beta$ gene transcription by the viral NS2A protein [5]; (d) blockade of phosphorylation of Tyk2 [2]; (e) down-regulation of IFNAR expression through virusinduced protein degradation pathways [92]; and (f) inactivation of the IFN-regulated JAK/ STAT signaling pathway [93] through redistribution of cellular cholesterol [94]. 

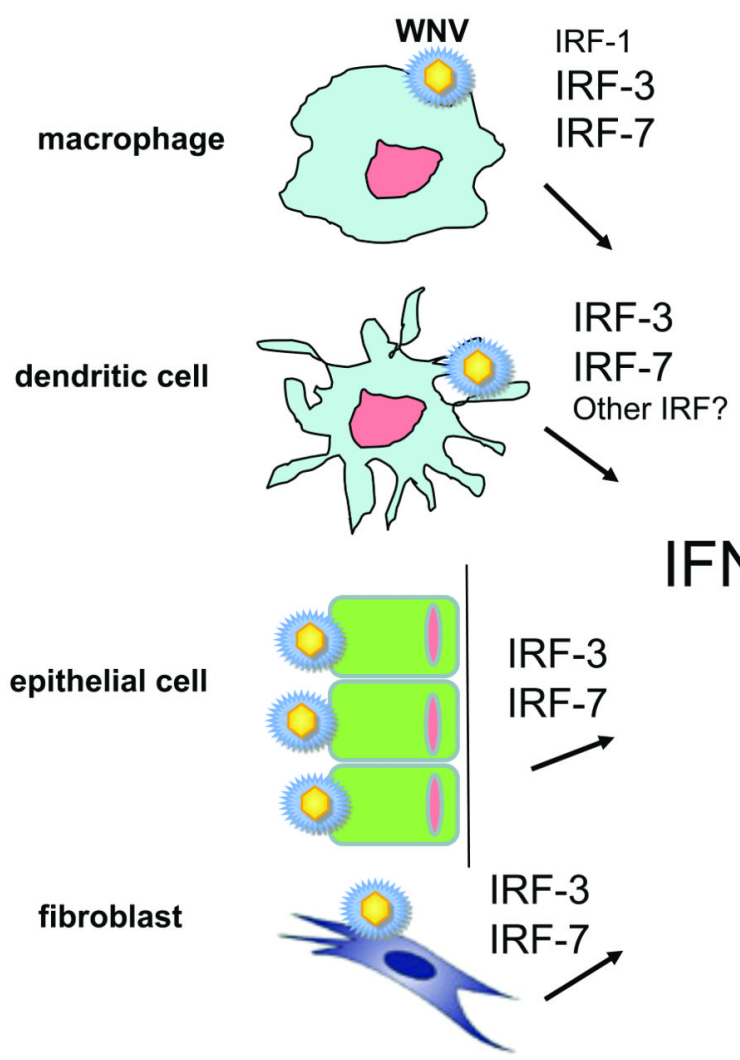

Figure 2. Induction of IFN- $\beta$ after WNV infection utilizes a different complement of IRF transcription factors in distinct cell types

In macrophages and $\mathrm{mDC}$, IFN- $\beta$ is induced at least partially through IPS-1-dependent yet IRF-3 and IRF-7-independent pathways. In macrophages FN- $\beta$ gene expression utilizes IRF-1 whereas in mDCs another transcription factor (possibly IRF-5) has a complementing role to IRF-3 and IRF-7. The smaller text size in the Figure suggests a subdominant role of these ancillary transcription factors compared to IRF-3 and IRF-7. In comparison, in epithelial cells and fibroblasts loss of IRF-3 and IRF-7 completely ablates IFN- $\beta$ gene induction after WNV infection. 
Table 1

List of ISGs with reported antiviral effector activity against infection by WNV and other flaviviruses

\begin{tabular}{|c|c|c|c|c|}
\hline ISG & Activity & $\begin{array}{l}\text { Mechanism of } \\
\text { Action }\end{array}$ & Flavivirus & Reference \\
\hline PKR & $\begin{array}{l}\text { Mice, primary } \\
\text { cells }\end{array}$ & $\begin{array}{l}\text { Translation } \\
\text { inhibition? } \\
\text { Induction of IFN }\end{array}$ & WNV & {$[44,47,71]$} \\
\hline RNAse L & $\begin{array}{l}\text { Mice, primary } \\
\text { cells }\end{array}$ & $\begin{array}{l}\text { Viral RNA } \\
\text { degradation? } \\
\text { Generation of } \\
\text { PAMP }\end{array}$ & WNV & {$[47,74]$} \\
\hline Oas1b & $\begin{array}{l}\text { Mice, primary } \\
\text { cells }\end{array}$ & $\begin{array}{l}\text { Reduction in } 2^{\prime}-5^{\prime} \\
\text { oligoA production }\end{array}$ & $\begin{array}{l}\text { WNV, other } \\
\text { flaviviruses }\end{array}$ & {$[75,76]$} \\
\hline Viperin (rsad2) & $\begin{array}{l}\text { Mice, primary } \\
\text { cells, ectopic } \\
\text { expression }\end{array}$ & $\begin{array}{l}\text { Modulation of } \\
\text { lipid biosynthesis } \\
\text { or droplet } \\
\text { formation? }\end{array}$ & $\begin{array}{l}\text { WNV, } \\
\text { Dengue }\end{array}$ & {$[71,83]$} \\
\hline IFIT1 & $\begin{array}{l}\text { Mice, primary } \\
\text { cells }\end{array}$ & $\begin{array}{l}\text { Translation } \\
\text { inhibition? }\end{array}$ & WNV & {$[3,89]$} \\
\hline IFIT2 & $\begin{array}{l}\text { Ectopic } \\
\text { expression }\end{array}$ & $\begin{array}{l}\text { Translation } \\
\text { inhibition? }\end{array}$ & WNV & [3] \\
\hline IFITM2 & $\begin{array}{l}\text { Ectopic } \\
\text { expression, } \\
\text { primary cells }\end{array}$ & $\begin{array}{l}\text { Inhibition of } \\
\text { entry? }\end{array}$ & $\begin{array}{l}\text { WNV, } \\
\text { Dengue }\end{array}$ & {$[71,90]$} \\
\hline IFITM3 & $\begin{array}{l}\text { Ectopic } \\
\text { expression, } \\
\text { primary cells }\end{array}$ & $\begin{array}{l}\text { Inhibition of } \\
\text { entry? }\end{array}$ & $\begin{array}{l}\text { WNV, } \\
\text { Yellow } \\
\text { fever }\end{array}$ & {$[66,71,87,90]$} \\
\hline ISG20 & $\begin{array}{l}\text { Ectopic } \\
\text { expression }\end{array}$ & Exonuclease? & $\begin{array}{l}\text { WNV, } \\
\text { Yellow } \\
\text { fever }\end{array}$ & {$[71,88]$} \\
\hline MB21D1 & $\begin{array}{l}\text { Ectopic } \\
\text { expression }\end{array}$ & Unknown & WNV & {$[66]$} \\
\hline HPSE & $\begin{array}{l}\text { Ectopic } \\
\text { expression }\end{array}$ & Unknown & $\begin{array}{l}\text { WNV, } \\
\text { Yellow } \\
\text { fever }\end{array}$ & {$[66]$} \\
\hline NAMPT & $\begin{array}{l}\text { Ectopic } \\
\text { expression }\end{array}$ & Unknown & WNV & {$[66]$} \\
\hline TRIM79a & $\begin{array}{l}\text { Ectopic } \\
\text { expression, } \\
\text { primary cells }\end{array}$ & $\begin{array}{l}\text { Degrades viral } \\
\text { RNA polymerase }\end{array}$ & $\begin{array}{l}\text { Tick-borne } \\
\text { encephalitis }\end{array}$ & [91] \\
\hline
\end{tabular}

Note, ISGs that inhibit flaviviruses other than WNV and their corresponding references are added for completeness but not necessarily discussed in the text. 